EPJ Web of Conferences 101, 02001 (2015)

DOI: 10.1051/epjconf/201510102001

(C) Owned by the authors, published by EDP Sciences, 2015

\title{
A planet in a polar orbit of 1.4 solar-mass star
}

Guenther, E.W. ${ }^{1, \text { a }}$, Cusano, F. ${ }^{2}$, Deeg, H..$^{3,4}$, Gandolfi, D. ${ }^{5}$, Geier, S. ${ }^{6}$, Grziwa, S. ${ }^{7}$, Heber, U. ${ }^{8}$, Tal-Or, L. ${ }^{9}$, Sebastian, D. ${ }^{1}$, Rodler, F. ${ }^{10,11}$, and the CoRoT-team

1 Thüringer Landessternwarte Tautenburg, Sternwarte 5, D-07778 Tautenburg, Germany

2 INAF-Osservatorio Astronomico di Bologna, Via Ranzani 1, I-40127 Bologna, Italy

3 Dpto. de Astrofísica, Universidad de La Laguna, E-38206 La Laguna, Tenerife, Spain

4 Instituto de Astrofísica de Canarias, E-38205 La Laguna, Tenerife, Spain

5 Zentrum für Astronomie Heidelberg, Landessternwarte Heidelberg-Königstuhl 12 D-69117 Heidelberg Germany

6 European Southern Observatory, Karl-Schwarzschild-Str. 2, D-85748, Garching, Germany

7 Rheinisches Institut für Umweltforschung an der Universiät zu Köln, Aachener Straße 209, D50931 Köln, Germany

8 Dr. Karl Remeis-Observatory \& ECAP, Astronomical Institute, Friedrich-Alexander University Erlangen-Nürnberg, Sternwartstr. 7, D-96049, Bamberg, Germany

9 School of Physics and Astronomy, Raymond and Beverly Sackler Faculty of Exact Sciences, Tel Aviv University, Tel Aviv, Israel

10 Max-Planck-Institut für Astronomie, Königstuhl 17, D-69117 Heidelberg, Germany

11 Harvard-Smithsonian Center for Astronomy, 60 Garden St., MA-02138 Cambridge, USA

\begin{abstract}
Although more than a thousand transiting extrasolar planets have been discovered, only very few of them orbit stars that are more massive than the Sun. The discovery of such planets is interesting, because they have formed in disks that are more massive but had a shorter life time than those of solar-like stars. Studies of planets more massive than the Sun thus tell us how the properties of the proto-planetary disks effect the formation of planets. Another aspect that makes these planets interesting is that they have kept their original orbital inclinations. By studying them we can thus find out whether the orbital axes planets are initially aligned to the stars rotational axes, or not. Here we report on the discovery of a planet of a 1.4 solar-mass star with a period of 5.6 days in a polar orbit made by CoRoT. This new planet thus is one of the few known close-in planets orbiting a star that is substantially more massive than the Sun.
\end{abstract}

\section{Introduction}

Although more than a thousand transiting extrasolar planets have been discovered, less than $4 \%$ of them orbit stars in the mass-range $M_{\text {star }}=1.3-2.1 M_{\odot}$. This means that we know only very little about planets around intermediate mass stars (IMSs) with short orbital periods. This lack of knowledge is unfortunate, because such planets can give us key information on how planets form and migrate.

Studies of giant stars and direct imaging surveys show that IMSs have a much higher frequency of massive planets than solar-like stars (Vigan et al. 2012 [20]; Johnson et al. 2010a [14]; Johnson et al. 2010b [15]). However, the life-time of the disks of these stars is on average about half as long as that of solar-like stars $\left(\tau_{\text {disk }}=1.2 \mathrm{Myrs}\right.$ for $M_{*} \geq 1.3 M_{\odot}$ compared to $\tau_{\text {disk }}=2.5$ Myrs for $M_{*} \sim 1 M_{\odot}$; Mamajek 2009 [16]). However, some Herbig AeBe stars can still have disks when they are older than 10 Myrs (Dent et al. 2013 [10]). The detection of a high frequency of planets orbiting IMSs means that

a e-mail: guenther@tls-tautenburg.de

This is an Open Access article distributed under the terms of the Creative Commons Attribution License 4.0, which permits unrestricted use, distribution, and reproduction in any medium, provided the original work is properly cited. 
planets can form within about one Myr. However, the direct imaging surveys and studies of giant stars are limited to planets at distances larger than $0.5 \mathrm{AU}$, and thus do not tell us, whether IMSs also have close-in planets. Whether they have close-in planets, or not, depends on the migration. By determining the frequency of close-in planets, we can thus constrain the time-scale of type II migration. If type II migration is fast, there should be many close-in planets of IMSs, if it is slow, there should be just a few. Based on data obtained for subgiants stars, Bowler et al. 2010 [3] concludes that IMSs have less close-in planets than solar-like stars. However, very few dedicated surveys for short-period planets of IMSs have so far been carried out. Currently, the best examples for such planets are WASP-33b, a planet of an 1.5 $M_{\text {Sun }}$-star at a distance of 0.026 AU (Collier Cameron et al. 2010 [6]), Kepler-13b, a planet of an 2.1 $M_{\text {Sun }}$-star at a distance of $0.036 \mathrm{AU}$ (Borucki et al. 2011 [2]), WASP-78b, a planet of an $2.0 M_{\text {Sun }}$-star at a distance of $0.042 \mathrm{AU}$ (Smalley et al. 2012 [18]), WASP-82b, a planet of an $1.6 \mathrm{M}_{\text {Sun }^{-}}$ star at a distance of $0.045 \mathrm{AU}$ (West et al. 2013 [21]), and HD102956b, a planet of an $1.7 M_{\text {Sun }}$ star at a distance of $0.081 \mathrm{AU}$ (Johnson et al. 2010b [15]).

Another interesting aspect of close-in planets of stars with $M_{\text {star }} \geq 1.2 M_{\odot}$ (corresponding to $T_{\text {eff }} \leq 6250 \mathrm{~K}$ ) is that they are often misaligned (Winn et al. 2010 [22]). It is generally assumed that misaligned systems are caused by dynamical interaction. Dynamical interaction like planet-planet scattering, secular evolution, and the Kozai mechanism can lead to large obliquities after the gas in the disk has been depleted (Xue et al. 2014 [23]). The obliquities observed for planets of IMSs thus indicate that at least these planets migrated because of dynamical interaction, rather than type II migration. By determining the distribution of the obliquities, it is thus possible to put constraints how planets migrate. Close-in planets of IMSs thus give us very important information on how long it takes for planets to form, and how they migrate. The determination of the frequency of close-in planets of IMSs thus tells us a lot about planet formation and migration. We have initiated a survey for close-in planets of IMSs using CoRoT-data, because a transit survey has the advantage that it is not biased against rapid rotation.

\section{CoRoT observations}

LRc07 E2 0307 (CoRoT ID: 652345526) is a $V=13.055 \pm 0.066$ mag star located at RA (2000.0) $18^{h} 31^{m} 00.241^{s}$, and Dec (2000.0) $+07^{\circ} 11^{\prime} 00.6^{\prime \prime}$. It was continuously observed with the CoRoT satellite from 2011 April 8 to June 28, thus over a period of 81.2 days. In total 218441 photometric measurements in three colours with a time-sampling is 32 seconds were obtained. Fifteen transits were observed with CoRoT. The orbital period is $5.616531 \pm 0.000023$ days, the epoch of the transit $B J D 2456864.459 \pm 0.005$, the duration $4.853 \pm 0.022 \mathrm{~h}$, and the depth $0.934 \pm 0.024 \%$.

\section{Ground-based observations}

Since the photometric mask of CoRoT has a size of about 30 " $\times 16$ ", we must confirm that the transit is not due to an eclipsing binary within the photometric mask. Removing such false-positives (FPs) is an important step in all planet search programs. The rate and nature of FPs, and the way how to detect and remove them is described in Almenara et al. 2009 [1]. To demonstrate that LRc07 E2 0307 is the transiting object, and not one of the stars close to it, we took images during transit ("on") and images out of transit ("off"). If the transit were caused by an unresolved, eclipsing binary within the photometric mask, this binary would be fainter in the image taken during transit than in the image taken out-of-transit (see Deeg et al. 2009 [7] for details on this method). We obtained three data-sets. One with the WISE-1-m-telescope, and two with the IAC-80-cm telescope. The quality of the data obtained is high enough to show the transit itself. This clearly shows that the transiting object is at least within two arcsec of the star. We also used these observations to improve the ephemeris.

Although it has been argued that the exclusion of FPs at distances larger than 1-2 arcsec is sufficient to show that the star is the transiting object, detailed studies show that AO-imaging is essential for confirming transiting planets, otherwise the probability for FPs is unacceptably high (Guenther et al. 2013 [13]). We thus obtained images with PISCES, the adaptive optics imager of the Large Binocular 


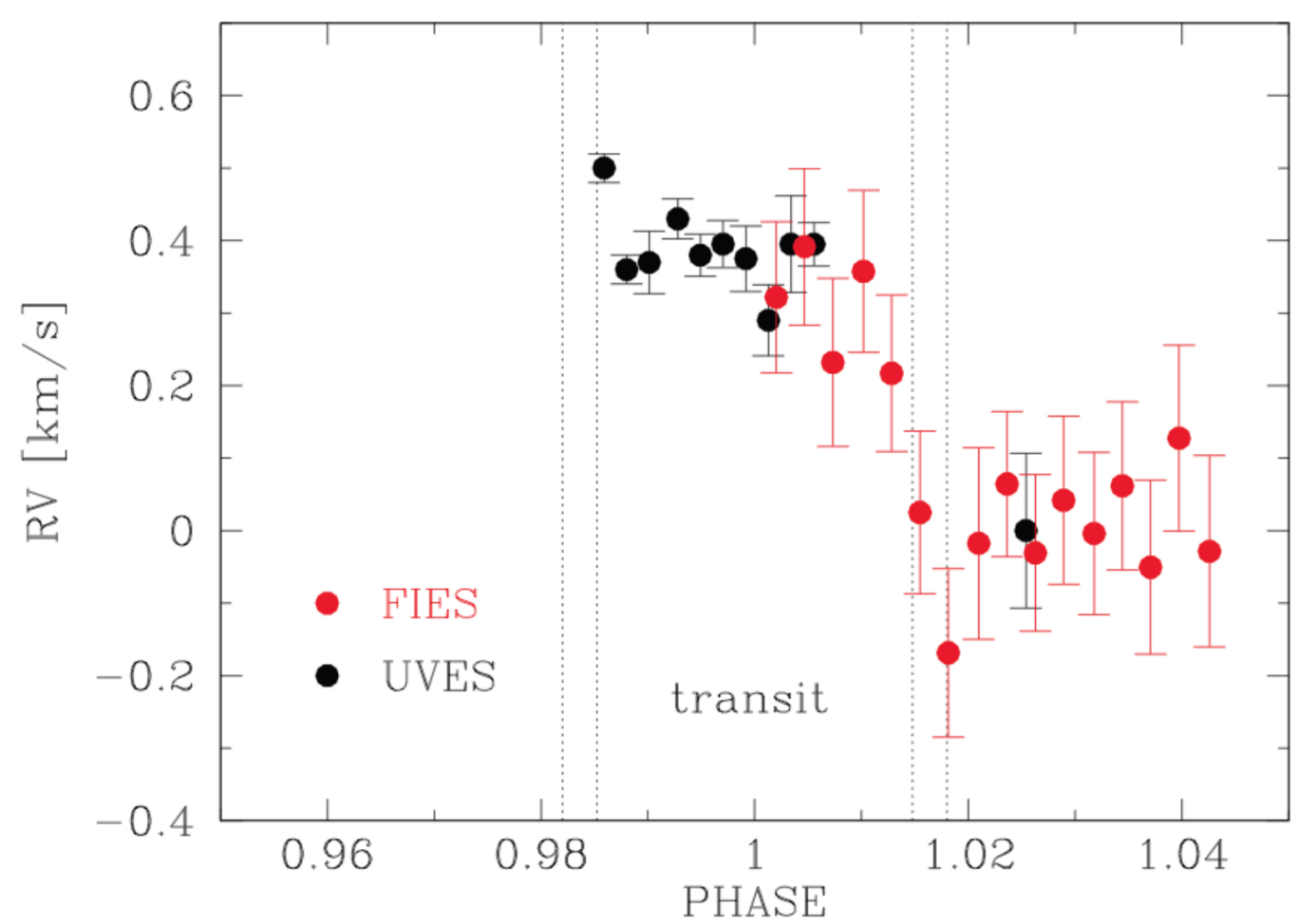

Fig. 1. Radial-velocity measurements taken during, and after the transit with UVES and FIES. During transit the radial-velocity is shifted with respect to the out-of-transit data indicating the planet has a polar orbit.

Telescope (LBT). From the depth of the transit, and the brightness of the star, we conclude that FPs have to be brighter than $\mathrm{V}=18.1 \pm 0.1 \mathrm{mag}$. However, the adaptive optics imaging was taken at infrared wavelengths. We thus have to estimate the brightness of potential FPs in the optical regime from their brightness in the infrared. Because of the extinction, faint background stars will be much brighter at infrared wavelength than in the optical. Faint stars in the foreground will also have red colours, because these will be late-type stars, and faint companions will also be brighter at infrared wavelength, because they must have a very late spectral type. Thus, in all cases, a FP will be brighter in the infrared than in the optical. We detected two previously unknown stars. Star No. 1 is $1.94 \pm 0.02^{\prime \prime} \mathrm{W}, 0.30 \pm 0.03^{\prime \prime}$ $\mathrm{S}$ from the target and has $J=15.7 \pm 0.1 \mathrm{mag}, K=16.2 \pm 0.1$, mag and star No. 2 is $3.42 \pm 0.02^{\prime \prime} \mathrm{E}$, $0.53 \pm 0.02^{\prime \prime} \mathrm{N}$, and has $J=17.1 \pm 0.1 \mathrm{mag}, K=16.8 \pm 0.1 \mathrm{mag}$. Both stars are not visible in the images taken with the WISE and IAC-80-cm telescopes, and both stars are much too faint to be FPs.

In order to determine the mass of the planet, we obtained 6 out-of-transit spectra of LRc07 E2 0307 with the HARPS spectrograph between June 15 and July 10 in 2012. From these spectra, we could only obtain an upper limit of $1.4 M_{J u p}$ for the planet. We also used the co-added HARPS spectra to determine the stellar parameters using the methods described in Valenti \& Piskunov (1996, [19]), Deleuil et al. (2008, [8]) and Bruntt et al. (2010, [5]). We find that the star has a spectral type F3VF5IV, $T_{\text {eff }}=6430 \pm 70 K$, and $\log g=4.0$. The mass of the host star is $1.43 \pm 0.04 M_{\odot}$ and the radius $1.93 \pm 0.01 R_{\odot}$. The age is $2.3 \pm 0.2$ Gyrs.

In order to confirm the planet, we obtained two sets of high-resolution spectra taken in- and out of transit. We obtained 6 spectra during transit, and 10 out of transit with the fibre-fed echelle spectrograph FIES, which is mounted on the $2.6 \mathrm{~m}$ Nordic Optical Telescope (NOT) at Roque de los Muchachos Observatory (La Palma, Spain). For these observations, we used the 1.3" high-resolution fibre, which gives a resolving power of $\lambda / \Delta \lambda \approx 67000$ and covers the wavelength range $360-740 \mathrm{~nm}$. The second data-set was obtained with UVES on ESO VLT UT-2 (KUEYEN). The transit was observed 
on the 6 August 2013. Each in-transit spectrum was exposed for 970s, the out of transit spectrum for 1450s. The in-transit observations were obtained for $2^{h} 55^{m}$, and the out-of-transit spectrum was taken $2^{h} 9^{m}$ after the end of in-transit observations in the same night. In this way we minimized the risk that changes of the instrument, or changes of features on the stellar surface affect the results. We used the standard 390+580 setting, which covers the wavelength region from 329 to $452 \mathrm{~nm}$ in the blue and 478 to $676 \mathrm{~nm}$ in the red arm. We used a slit-width of $0.8 \mathrm{arcsec}$, which provides a resolution of about $\lambda / \Delta \lambda=61000$. Thus, there are 4 pixels per resolution element. The $\mathrm{S} / \mathrm{N}$ at $600 \mathrm{~nm}$ is on average 240 per resolution element. Both data-sets show the shift of $0.4 \mathrm{~km} / \mathrm{s}$ during transit with respect to the outof-transit spectra indicating the planet to have a polar orbit (Fig. 1). We also used the UVES spectra to exclude an unresolved FP in a similar way as described in Guenther and Tal-Or 2010 [11]. We thus finally conclude that LRc07 E2 0307 has a planet with $M_{p}<1.4 M_{J u p}$ and $R_{p}=1.9 R_{J u p}$.

\section{Discussion and conclusions}

Using the information about CoRoT-targets in the EXODAT-catalog, we find that CoRoT has observed 125874 stars in the long runs of which about 14\% are IMSs (Deleuil et al. 2009 [9]). This number agrees reasonably well with the frequency of F5V to A5V-stars determined from spectroscopy for the IRa01, LRa01, LRa02, and LRa06 fields (Guenther et al. 2012 [12], Sebastian et al. 2012 [17]). If the frequency of planets of IMSs were the same as that of low-mass stars, we expect to find about $13 \pm 7$ planets of IMSs in the CoRoT sample. Since CoRoT has discovered six planets and brown dwarfs already, there could be still up to seven planets of IMSs in the CoRoT-database. LRc07 E2 0307 seems to be one of them. We will continue the survey for planets of IMSs with CoRoT until the remaining candidates are solved. However, as up to now, we can already state that the frequency of close-in planets of IMSs seems to be equal, or smaller than that for solar-like stars.

\section{References}

1. Almenara, J. M., Deeg, H. J., Aigrain, S., et al. 2009, A\&A, 506, 337

2. Borucki, W. J., Koch, D. G., Basri, G. et al. 2011, ApJ, 736, 19

3. Bowler, B.P., Johnson, J.A., Marcy, G.W., et al. 2010, ApJ, 709, 396

4. Bruntt, H.,Catala, C., Garrido, R., et al. 2002, A\&A, 389, 345

5. Bruntt, H., Deleuil, M., Fridlund, M., et al. 2010, A\&A, 519, A51

6. Collier Cameron, A., Guenther, E., Smalley, B. et al. 2010, MNRAS, 407, 507

7. Deeg, H.J. et al. 2009, A\&A, 506, 343

8. Deleuil, M., Deeg, H. J., Alonso, R., et al. 2008, A\&A, 491, 889

9. Deleuil, M., Meunier, J.C., Moutou, C., et al. 2009, AJ, 138, 649

10. Dent, W.R.F., Thi, W.F., Kamp, I., et al. 2013, PASP, 125, 477

11. Guenther, E.W., \& Tal-Or, L. 2010, A\&A, 521, AA83

12. Guenther, E. W., Gandolfi, D., Sebastian, D., et al. 2012, A\&A, 543, A125

13. Guenther, E. W., Fridlund, M., Alonso, R., et al. 2013, A\&A, 556, AA75

14. Johnson, J.A., Howard, A.W., Bowler, B.P., et al. 2010a, PASP, 122, 701

15. Johnson, Johnson, J.A., Bowler, B.P., Howard, A.W., et al. 2010b, ApJlet, 721, L153

16. Mamajek, E.E. 2009, American Institute of Physics Conference Series, 1158, 3

17. Sebastian, D., Guenther, E. W., Schaffenroth, V., et al. 2012, A\&A, 541, A34

18. Smalley, B., Anderson, D. R., Collier-Cameron, A., et al. 2012, A\&A, 547, AA61

19. Valenti, J. A., \& Piskunov, N. 1996, A\&Asup, 118, 595

20. Vigan, A. et al 2012 A\&A 544, 9

21. West, R. G., Almenara, J.-M., Anderson, D. R., et al. 2013, arXiv:1310.5607

22. Winn, J.N., Fabrycky, D., Albrecht, S., \& Johnson, J. A. 2010, ApJlet, 718, L145

23. Xue, Y., Suto, Y., Taruya, A., et al. 2014, ApJ, 784, 66 